\title{
The Hydrological Cycle over a Wide Range of Climates Simulated with an Idealized GCM
}

\author{
Paul A. O'Gorman and Tapio Schneider \\ California Institute of Technology, Pasadena, California
}

(Manuscript received 29 May 2007, in final form 10 December 2007)

\begin{abstract}
A wide range of hydrological cycles and general circulations was simulated with an idealized general circulation model (GCM) by varying the optical thickness of the longwave absorber. While the idealized GCM does not capture the full complexity of the hydrological cycle, the wide range of climates simulated allows the systematic development and testing of theories of how precipitation and moisture transport change as the climate changes. The simulations show that the character of the response of the hydrological cycle to variations in longwave optical thickness differs in different climate regimes.

The global-mean precipitation increases linearly with surface temperature for colder climates, but it asymptotically approaches a maximum at higher surface temperatures. The basic features of the precipitation-temperature relation, including the rate of increase in the linear regime, are reproduced in radiativeconvective equilibrium simulations. Energy constraints partially account for the precipitation-temperature relation but are not quantitatively accurate.

Large-scale condensation is most important in the midlatitude storm tracks, and its behavior is accounted for using a stochastic model of moisture advection and condensation. The precipitation associated with large-scale condensation does not scale with mean specific humidity, partly because the condensation region moves upward and meridionally as the climate warms, and partly because the mean condensation rate depends on isentropic specific humidity gradients, which do not scale with the specific humidity itself.

The local water vapor budget relates local precipitation to evaporation and meridional moisture fluxes, whose scaling in the subtropics and extratropics is examined. A delicate balance between opposing changes in evaporation and moisture flux divergence holds in the subtropical dry zones. The extratropical precipitation maximum follows the storm track in warm climates but lies equatorward of the storm track in cold climates.
\end{abstract}

\section{Introduction}

Study of the hydrological cycle of altered climates has focused on climates with increased carbon dioxide concentrations such as are expected to occur in the coming decades and centuries (e.g., Manabe and Stouffer 1980; Boer 1993; Hennessy et al. 1997; Allen and Ingram 2002; Held and Soden 2006; Meehl et al. 2007) and at the last glacial maximum and other earlier climates (e.g., Manabe and Broccoli 1985; Broccoli 2000; Pierrehumbert 2002). Here we simulate a wide range of hydrological cycles and general circulations with an idealized general circulation model (GCM) to

Corresponding author address: Paul O’Gorman, California Institute of Technology, Mail Code 100-23, 1200 E. California Blvd., Pasadena, CA 91125.

E-mail: pog@caltech.edu systematically develop and test theories of how precipitation and moisture transport change as the climate changes.

We address how the global-mean precipitation, large-scale condensation, poleward moisture flux, and local precipitation change as the optical thickness of the longwave absorber changes. Because of the strong dependence of saturation specific humidity on temperature in the Clausius-Clapeyron relation and because the relative humidity is not expected to change greatly as climate forcings change (Held and Soden 2000), the mean specific humidity is very sensitive to changes in longwave optical thickness. However, it is well known that in simulations of global warming, the global-mean precipitation does not change in proportion to the amount of water vapor in the atmosphere (e.g., Allen and Ingram 2002; Held and Soden 2006). It has been proposed that changes in latent heat release in the tro- 
posphere are balanced by changes in longwave radiative cooling and that changes in longwave cooling and thus in precipitation do not keep pace with changes in mean specific humidity (Allen and Ingram 2002). Such energy constraints on precipitation are attractive because they tie changes in global-mean precipitation to changes in radiative fluxes without the need for detailed knowledge of precipitation processes. However, their adequacy is unclear and is assessed here (section 3).

In addition to considerations of the global energy budget, mechanistic considerations of water vapor transport and condensation are also helpful for understanding changes in the hydrological cycle. We analyze the large-scale condensation associated with baroclinic eddies in the extratropics using a stochastic model of moisture transport and condensation and study how precipitation arising from large-scale condensation (large-scale precipitation) changes with climate (section 4). In particular, we address the question of how the extratropics adjust so that changes in large-scale precipitation do not keep pace with changes in mean specific humidity.

Global energy constraints do not apply to local precipitation, for example, in subtropical dry zones or midlatitude storm-track regions. Instead, we use the column water vapor budget to relate the local precipitation rate to the local evaporation rate and meridional moisture flux divergence. The meridional moisture flux is important because of its implications for local precipitation and evaporation and because it represents a latent heat flux (section 5). In a warmer climate, both mean and eddy moisture fluxes can be expected to increase (e.g., Held and Soden 2006). We use scalings for the divergence of the mean and eddy moisture fluxes to help explain changes in the intensity of the pattern of evaporation minus precipitation (section 6). In terms of societal impact, spatial shifts in the location of extrema of the mean precipitation distribution may be equally important and are also discussed.

The simulated climates are sampled from a continuum of equilibrium climates defined by changes in longwave optical thickness. This is similar in spirit to the study by Caballero and Langen (2005) of the meridional latent heat flux in a series of simulations with different imposed sea surface temperature distributions, but we use a mixed layer ocean with a closed energy budget as the lower boundary in order to obtain energetically consistent changes in precipitation. We use an idealized GCM based on the Geophysical Fluid Dynamics Laboratory (GFDL) dynamical core with simplified moist parameterizations (Frierson 2007). We represent the radiative effects of changes in both water vapor and well-mixed greenhouse gases such as carbon dioxide by changing the total longwave optical thickness in a two-stream gray radiation scheme. Questions related to cloud effects or to precipitation statistics other than mean quantities (e.g., precipitation extremes) are postponed, and we focus on arguments that are less parameterization- or resolution-dependent. A theme of our study is the extent to which the hydrological cycle responds linearly to climate changes and whether different responses can be expected in different climate regimes. This is important when we attempt to use our knowledge of the present-day hydrological cycle and its variations to understand the hydrological cycle in very different climates.

Some observational studies of the precipitation changes of recent decades have found that global and tropical precipitation vary more strongly in observations than in climate simulations (Wentz et al. 2007; Allan and Soden 2007), but it is not clear whether there is a significant longer-term trend in global-mean precipitation (Gu et al. 2007). Our results provide a framework in which these and other precipitation changes can be understood and evaluated.

\section{Idealized GCM}

We use a moist idealized GCM similar in many aspects to that of Frierson et al. (2006) and Frierson (2007). ${ }^{1}$ The use of a consistent surface energy budget is key for this study in which energy constraints on evaporation play an important role. Therefore, we use a two-stream gray radiation scheme rather than Newtonian cooling, which is often used in dry idealized GCMs (Held and Suarez 1994; Schneider 2004), but which makes the inclusion of a surface energy budget difficult. The lower boundary is a mixed layer ocean of depth $1 \mathrm{~m}$ that does not transport heat horizontally. The resulting climates are statistically stationary and axisymmetric.

The longwave optical thickness is specified by $\tau=$ $\alpha \tau_{\text {ref }}$, with the optical thickness of a reference simulation, $\tau_{\text {ref }}$, given by

$$
\tau_{\text {ref }}=\left[f_{l} \sigma+\left(1-f_{l}\right) \sigma^{4}\right]\left[\tau_{e}+\left(\tau_{p}-\tau_{e}\right) \sin ^{2} \phi\right],
$$

where $f_{l}=0.2, \sigma=p / p_{s}$ is pressure $p$ normalized by surface pressure $p_{s}, \phi$ is latitude, and the longwave op-

\footnotetext{
${ }^{1}$ Our GCM differs from that described in Frierson et al. (2006) and Frierson (2007) by using a Monin-Obukhov scheme that allows for an unstable boundary layer, by not including reevaporation in the large-scale condensation scheme, by not approximating the relation between specific humidity and vapor pressure, by having a different variant of the convection scheme, and by several differences in parameter values.
} 
tical thicknesses at the equator and at the pole are $\tau_{e}=7.2$ and $\tau_{p}=1.8$, respectively. The longwave optical thickness is varied by setting the factor $\alpha$ to a different value in each of a series of 16 simulations, with $0.2 \leq \alpha \leq 6.0$.

The longwave optical thickness does not depend on the water vapor field, so we are not allowing for radiative water vapor feedback or cloud feedbacks. The changes in imposed longwave optical thickness should be taken as an idealized representation of changes in all longwave absorbers, including carbon dioxide and water vapor. The absence of radiative water vapor feedback in our simulations is not a severe limitation so long as we restrict ourselves to questions regarding the response of the hydrological cycle to changes in total longwave absorber optical thickness rather than to changes in the concentration of one particular greenhouse gas.

The top-of-atmosphere (TOA) insolation is imposed as a perpetual equinox with no diurnal cycle,

$$
S_{\mathrm{TOA}}=\frac{S_{0}}{4}\left[1+\frac{\Delta_{s}}{4}\left(1-3 \sin ^{2} \phi\right)\right],
$$

where $S_{0}=1360 \mathrm{~W} \mathrm{~m}^{-2}$ and $\Delta_{s}=1.2$. Absorption of solar radiation in the atmosphere is imposed such that the downward shortwave flux at a given sigma level and latitude is

$$
S=S_{\mathrm{TOA}} \exp \left[-\tau_{s} \sigma^{2}\right],
$$

where $\tau_{s}=0.22$. Changes in shortwave absorption owing to changes in water vapor concentrations in the atmosphere are not taken into account for simplicity, although they may play a significant role, particularly in warmer climates. The surface albedo is constant at $a=$ 0.38 , with all reflected solar radiation escaping directly to space.

A simple quasi-equilibrium scheme is used to parameterize moist convection by relaxation of temperatures toward a moist adiabat and of specific humidities toward a profile with a reference relative humidity of $70 \%$, with a 2 -h relaxation time for both temperature and specific humidity. The convection scheme is the same as that described in Frierson (2007) with the "shallower" shallow convection option, except for one difference in how enthalpy conservation is enforced. In the case of deep convection in which the enthalpy change resulting from the specific humidity adjustment is greater than that from the temperature adjustment, the time scale for the specific humidity adjustment is lengthened so that enthalpy is conserved at each time step (D. M. W. Frierson 2006, personal communication). This variant of the convection scheme results in less extratropical convective precipitation compared with the scheme described in Frierson (2007), where a shift in the reference temperature profile is used to conserve enthalpy in all deep-convective cases.

A large-scale (grid scale) condensation parameterization ensures that the relative humidity in a grid cell does not exceed $100 \%$. To facilitate comparison with stochastic models of water vapor advection and condensation (Pierrehumbert et al. 2007; O'Gorman and Schneider 2006), there is no reevaporation of falling condensate.

We only consider the vapor-liquid phase change and compute the saturation vapor pressure $e_{s}$ from a simplified Clausius-Clapeyron relation with constant latent heat of vaporization $L$,

$$
e_{s}(T)=e_{0} \exp \left[-\frac{L}{R_{v}}\left(\frac{1}{T}-\frac{1}{T_{0}}\right)\right]
$$

with $R_{v}=461.5 \mathrm{~J} \mathrm{~kg}^{-1} \mathrm{~K}^{-1}, L=2.5 \times 10^{6} \mathrm{~J} \mathrm{~kg}^{-1}, e_{0}=$ $610.78 \mathrm{~Pa}$, and $T_{0}=273.16 \mathrm{~K}$. We take account of the effect of water vapor on the density of air (virtual temperature effect). ${ }^{2}$

Bulk aerodynamic formulae are used for the surface fluxes with an additive gustiness term of $1 \mathrm{~m} \mathrm{~s}^{-1}$ in surface velocities. Drag coefficients are calculated using Monin-Obukhov similarity theory. The roughness length is $5 \times 10^{-3} \mathrm{~m}$ for momentum and $10^{-5} \mathrm{~m}$ for moisture and sensible heat. Boundary layer turbulence is parameterized using a $k$-profile scheme, similar to that of Troen and Mahrt (1986), with a dynamically determined boundary layer height.

The primitive equations were solved spectrally at $\mathrm{T} 42$ resolution with 30 unevenly spaced sigma levels in the vertical and with $\nabla^{8}$ hyperdiffusion of vorticity, divergence, and temperature. Water vapor is advected by a finite-volume scheme at an equivalent resolution. Simulations were either spun up over 700 days from an isothermal rest state or over 200 days starting from the end state of a simulation with a similar longwave optical thickness. Time averages were taken over a subsequent 300-day period.

Figure 1 shows the mean potential temperature, streamfunction of the mean meridional circulation, and mean relative humidity for three simulations with $\alpha=0.2,1$, and 6 . The reference simulation $(\alpha=1)$ has an earthlike climate, with a global-mean surface air temperature (temperature of the lowest model level) of $288 \mathrm{~K}$. The Hadley cells are stronger than earth's annual-mean Hadley cells, partially because of the absence of oceanic meridional heat fluxes in the simula-

\footnotetext{
${ }^{2}$ Changes in the mass of the atmospheric column due to precipitation or evaporation are not taken into account in this GCM.
} 


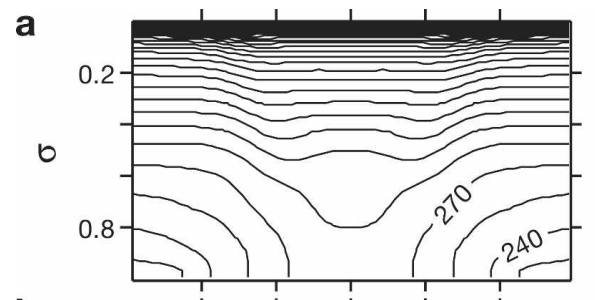

b

C

○
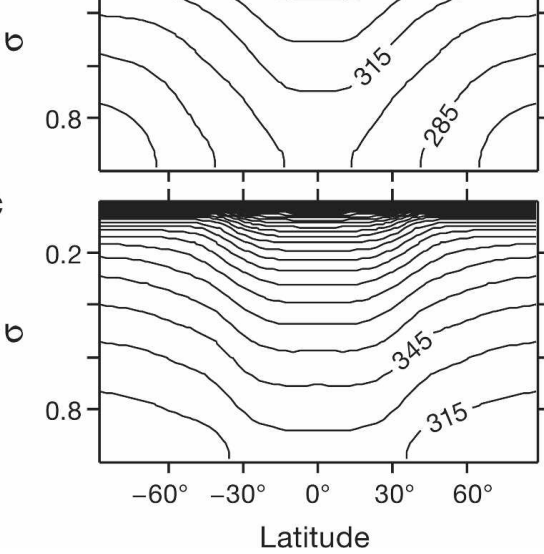
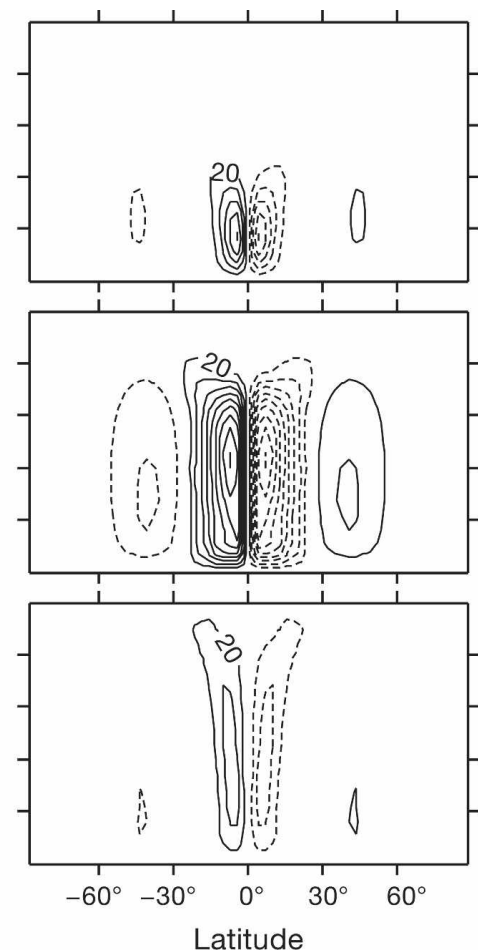

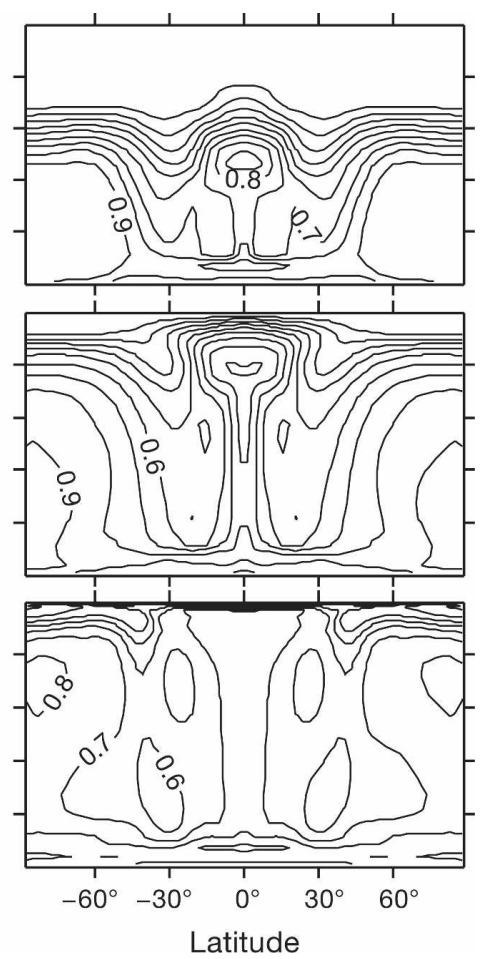

FIG. 1. Mean potential temperature, mass streamfunction, and relative humidity (zonal, time, and interhemispheric average) of (a) the coldest simulation $(\alpha=0.2)$, (b) the reference simulation $(\alpha=1)$, and (c) the warmest simulation $(\alpha=6)$ : (left) Potential temperature (contour interval $15 \mathrm{~K}$ ), (middle) mass streamfunction (contour interval $20 \times 10^{9} \mathrm{~kg} \mathrm{~s}^{-1}$, negative contours dashed), (right) relative humidity (contour interval 0.1 ).

tions. The vertical extent of the Hadley cell can be seen to increase as the tropopause rises with increasing surface temperature. The difference in surface temperature between pole and equator decreases with increasing surface temperature over the range of climates simulated. For example, the reference simulation has an equator-to-pole difference in surface air temperature of $46 \mathrm{~K}$, whereas the simulation with $\alpha=2$ has a relatively equable climate, with an equator-to-pole difference in surface air temperature of $31 \mathrm{~K}$ (equatorial and polar temperatures of 311 and $280 \mathrm{~K}$, respectively).

The extratropical relative humidity in the lower free troposphere decreases as the surface temperature increases, while at upper levels it increases because of the rise in the tropopause (Fig. 1). Mean relative humidity remains relatively constant in simulations of global warming compared with much larger changes in specific humidity (Held and Soden 2000), but it need not stay exactly constant. Large-scale processes are thought to control the relative humidity distribution in the free troposphere outside of convective regions (e.g., Pierrehumbert and Roca 1998; Dessler and Sherwood 2000; Galewsky et al. 2005). Dehumidification by increasingly active convection may contribute to the decrease in extratropical relative humidity in the lower free tropo- sphere as climate warms in the simulations. We performed two alternate sets of simulations to test the importance of the specific choice of convection scheme for changes in relative humidity: one with no convection scheme and one with a higher reference relative humidity $(90 \%)$ in the convection scheme. While different from the simulations presented here in several respects, they also show a decrease in extratropical relative humidity in the lower free troposphere with increasing surface temperature.

Figure 2 shows the distribution of mean precipitation with latitude for the reference simulation, with a decomposition into precipitation parameterized as convection and as large-scale condensation. The globalmean precipitation is $4.3 \mathrm{~mm}$ day $^{-1}$, compared with 2.7 $\mathrm{mm}$ day $^{-1}$ observed for the annual-mean earth climate (Xie and Arkin 1997). The greater precipitation in the simulation can be partly attributed to the lower boundary condition since ocean covers the entire planet surface, but the idealized nature of the radiation scheme also contributes to the discrepancy. Precipitation associated with convection dominates in the tropics; largescale condensation becomes more important farther poleward. The midlatitude storm tracks and the arid subtropics are clearly recognizable. 


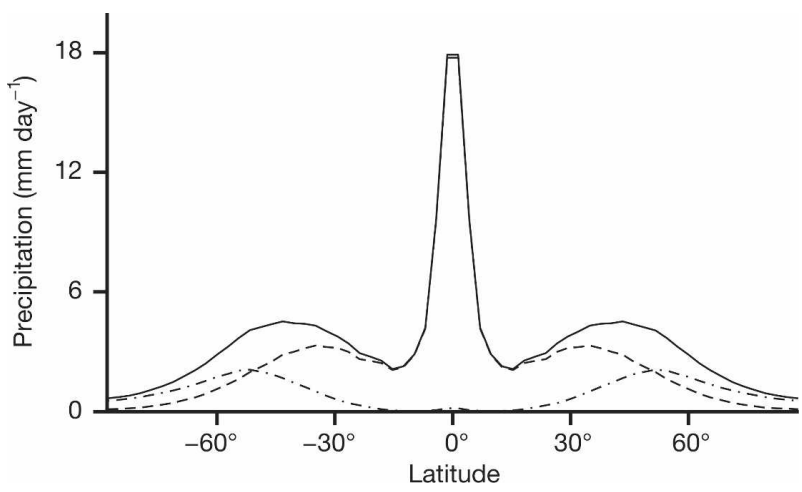

FIG. 2. Mean precipitation (zonal, time, and interhemispheric average) in the reference simulation (solid line) and decomposition into precipitation parameterized as convection (dashed line) and large-scale condensation (dash-dotted line).

\section{Global precipitation and energy constraints}

Figure 3 shows the global-mean precipitation as a function of global-mean surface air temperature. Each circle represents the equilibrium climate state for a particular longwave optical thickness. The precipitation increases monotonically with surface temperature, with two notable features: (i) at lower surface temperatures the precipitation increases linearly with temperature at a rate of $0.12 \mathrm{~mm} \mathrm{day}^{-1} \mathrm{~K}^{-1}$ and (ii) at higher surface temperatures the precipitation saturates, reaching 5.90 $\mathrm{mm}$ day $^{-1}$ in the warmest simulation. The reference simulation has a surface temperature close to that of present-day earth and lies near the edge of the linear response regime, where the precipitation begins to roll off to its maximum value. In the vicinity of the reference simulation, the fractional rate of increase of precipitation with respect to surface temperature is $2.5 \%$ $\mathrm{K}^{-1}$, which is slightly lower than the linear rate of increase of $0.12 \mathrm{~mm}$ day ${ }^{-1} \mathrm{~K}^{-1}$ shown as a dashed line in Fig. 3. This may be compared with an ensemble-mean increase of precipitation with surface temperature of order $2 \% \mathrm{~K}^{-1}$ relative to the present-day climate in comprehensive coupled climate simulations (Held and Soden 2006).

\section{a. Maximum global-mean precipitation and surface energy budget}

The saturation of global-mean precipitation at high surface temperatures can be understood from the surface energy budget at equilibrium,

$$
(1-a) S_{\mathrm{sfc}}-L P-R-F=0,
$$

where $(1-a) S_{\text {sfc }}$ is the absorbed shortwave radiative flux at the surface, $P$ is the precipitation, $R$ is the net upward longwave radiative flux at the surface, and $F$ is

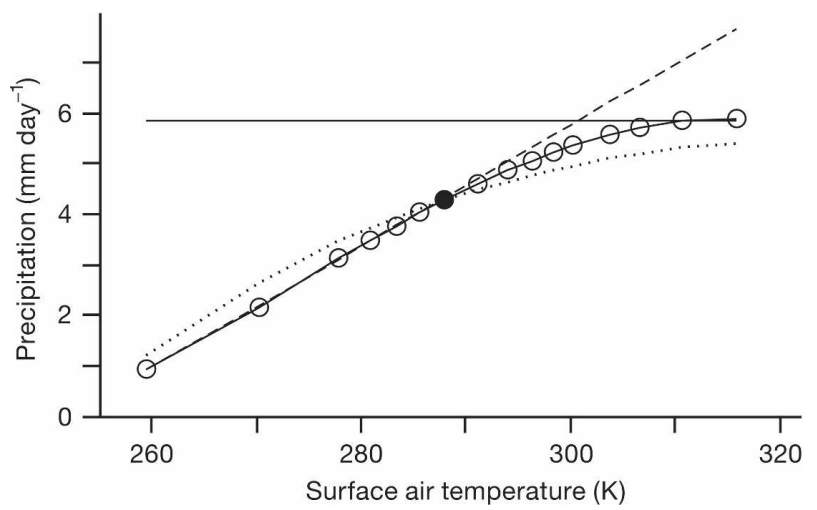

FIG. 3. Global-mean precipitation vs global-mean surface air temperature (solid line with circles). Here, and in the following figures, the reference simulation is shown with a filled circle. The dashed line passes through the coldest simulation and represents a linear rate of increase of $0.12 \mathrm{~mm} \mathrm{day}^{-1} \mathrm{~K}^{-1}$. The horizontal solid line is the constant precipitation of $5.85 \mathrm{~mm} \mathrm{day}^{-1}$ given by the approximation (6). The dotted line shows the approximation (9) of the global-mean precipitation using the longwave radiative loss of the atmosphere, with a constant offset chosen such that there is agreement with the global-mean precipitation for the reference simulation. The rate of increase at the reference simulation is $2.5 \% \mathrm{~K}^{-1}$ for precipitation and $1.6 \% \mathrm{~K}^{-1}$ for the longwave-loss approximation.

the net upward sensible heat flux at the surface. A time and global mean is implied for all fluxes, and we have used the fact that, at equilibrium, evaporation is equal to precipitation.

At high temperatures, the evaporation is much larger than the net surface longwave radiative flux or the sensible heat flux, and thus as a first approximation we can estimate a maximum precipitation,

$$
P_{\text {max }} \simeq(1-a) S_{\mathrm{sfc}} / L,
$$

which is $5.85 \mathrm{~mm} \mathrm{day}^{-1}$ in our simulations and is shown as the horizontal solid line in Fig. 3. This limit corresponds to all shortwave radiation absorbed at the surface being used to evaporate water. The precipitation saturates at a value somewhat higher than that given by the approximation (6) because, as the climate warms, the surface temperature disequilibrium (sea surface temperature minus surface air temperature) decreases, and the surface sensible heat flux becomes directed into the surface. The surface temperature disequilibrium is controlled by the surface energy budget and adjusts as the climate warms so that the surface latent heat flux can be balanced by the other surface fluxes despite large increases in saturation specific humidity. The boundary layer turbulent diffusivities decrease as the boundary layer becomes increasingly statically stable with increasing surface temperature. Consistent with the radiative-convective equilibrium simulations of 
Pierrehumbert (2002), we find that the asymptotic value of global-mean precipitation at high temperatures in our GCM is lower in an alternate set of simulations in which the increased buoyancy of moist air due to the virtual temperature effect is not taken into account. Another alternate set of simulations in which the longwave radiation scheme included a transparent spectral window (cf. Weaver and Ramanathan 1995) had a maximum global-mean precipitation significantly lower than that given by the approximation (6) because the window allowed the longwave cooling of the surface to remain larger at higher temperatures. In general, the exact asymptotic behavior of the precipitation in warm climates will depend on radiative transfer properties such as the presence of a spectral window for longwave radiation and the absorption of shortwave radiation by water vapor.

\section{b. Global-mean precipitation and longwave radiative loss of the atmosphere}

It has been argued (e.g., Allen and Ingram 2002) that the energy budget of the atmosphere (or troposphere) constrains changes in precipitation and, in particular, that changes in global-mean precipitation are primarily balanced by changes in the longwave radiative loss of the atmosphere. However, the longwave radiative loss of the atmosphere may not be a useful a priori constraint on the global-mean precipitation for several reasons. First, changes in surface sensible heat fluxes or shortwave radiation absorbed in the atmosphere may not be negligible. Second, the longwave radiative loss of the atmosphere depends on the net longwave flux at the surface and thus not only on the thermal structure of the atmosphere but also on the sea surface or land temperature. Changes in the difference between the surface air temperature and the sea surface or land temperature are themselves strongly linked to the evaporation through the surface energy budget, rendering any relation between changes in precipitation and longwave radiative loss implicit. Here, we are in a position to systematically test the validity of the longwave radiative loss argument.

The equilibrium energy budget of the atmosphere may be written

$$
L P+S_{\text {abs }}-R_{\text {loss }}+F=0,
$$

where $S_{\text {abs }}$ is the shortwave radiative flux absorbed in the interior of the atmosphere and $R_{\text {loss }}$ is the net longwave radiative loss of the atmosphere, including both the outgoing longwave radiation (OLR) and the net longwave flux at the surface. The perturbation energy budget between two equilibrium climates is

$$
L \delta P+\delta R+\delta F=0,
$$

where $R$ is the net upward surface flux of longwave radiation, and we have used the fact that, in our simulations, the OLR remains constant between different equilibrium climates and the latent heat of vaporization is constant. The perturbation energy budget of the atmosphere is equivalent to that of the surface [cf. Eq. (5)] in our simulations, but this is not generally the case. Neglecting changes in surface sensible heat flux, we find

$$
\delta P=-\delta R / L,
$$

as suggested by Allen and Ingram (2002).

The dotted line in Fig. 3 shows the approximation (9) in comparison with the actual precipitation. While it reproduces the qualitative dependence of precipitation on surface temperature, it is not accurate and, importantly, does not give the correct rate of change of precipitation near the reference simulation $\left(1.6 \% \mathrm{~K}^{-1}\right.$ for the approximation compared with $2.5 \% \mathrm{~K}^{-1}$ for the actual precipitation). The error arises because changes in surface sensible heat flux are not negligible. The surface sensible heat flux may be relatively small in magnitude, but it changes sign as the longwave optical thickness is varied and plays an important role in the perturbation energy budget. The dependence of precipitation on surface temperature is more linear than that of longwave radiative loss, so we cannot explain the linearity of the precipitation response with this approximation.

\section{c. Radiative-convective equilibrium simulations}

We have seen that simple energy constraints alone do not account for the behavior of the global-mean precipitation. We next examine if radiative-convective simulations exhibit a similar dependence of precipitation on surface temperature as the full dynamical simulations, since energy constraints should also operate in the radiative-convective simulations, and surface sensible heat fluxes can be included.

There have been several studies of precipitation in radiative-convective equilibrium (e.g., Renno et al. 1994; Pierrehumbert 2002). Here we make as direct a comparison as possible between our radiative-convective and full dynamical simulations by performing column radiative-convective simulations over the entire globe with the same radiative parameters as in the full dynamical simulations. There is no mean circulation or lateral transport between different atmospheric columns. Instantaneous wind fields are used in the full dynamical simulations to calculate surface fluxes and the boundary-layer height and diffusivities. Since the radiative-convective simulations do not calculate a wind field, we use the mean wind field from the final 


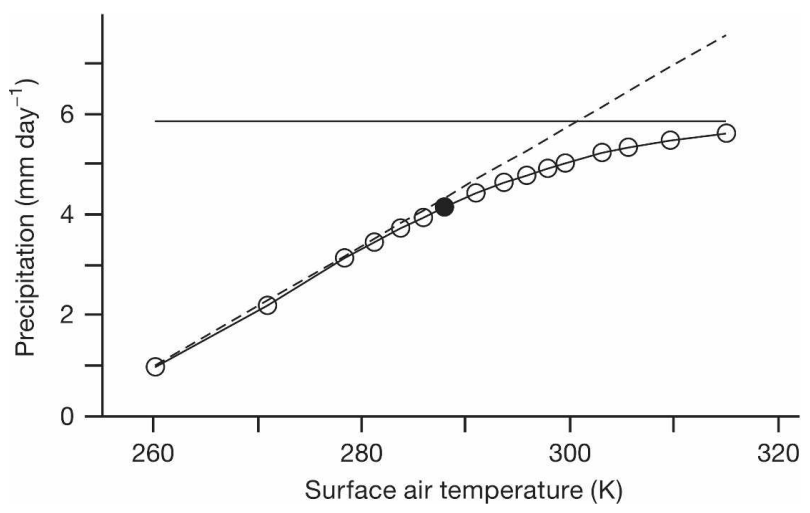

FIG. 4. Global-mean precipitation vs global-mean surface air temperature (solid line with circles) in the radiative-convective equilibrium simulations. To facilitate comparison, the dashed line and horizontal solid line are as in Fig. 3.

100 days of the reference dynamical simulation as an input to the surface-flux and boundary-layer schemes. Using the mean wind fields of the corresponding dynamical simulations in each case (rather than those of the reference simulation) gives qualitatively similar results. The radiative-convective simulations are axisymmetric, but not steady, with oscillations that have largest amplitude in the warmest simulations.

Figure 4 shows the resulting global-mean precipitation as a function of surface temperature. The precipitation is generally smaller than in the dynamical simulations, but the general character of the precipitationtemperature relation is similar. This may be because the global-mean precipitation is dominated by the contribution from the tropics, where radiative-convective equilibrium is a reasonable first approximation. The fractional rate of increase of precipitation with respect to surface temperature is $2.3 \% \mathrm{~K}^{-1}$ at the reference simulation compared with $2.5 \% \mathrm{~K}^{-1}$ for the full dynamical simulations, suggesting that the radiativeconvective equilibrium model captures the physics behind the rate of increase. This would represent a conceptual simplification and may be useful for model comparisons.

\section{d. Global-mean precipitation and column water vapor}

Global warming simulations exhibit a canonical $7 \%$ $\mathrm{K}^{-1}$ rise in atmospheric water vapor content relative to present-day values, consistent with Clausius-Clapeyron scaling for the saturation specific humidity and an assumption of constant relative humidity (Trenberth et al. 2003; Held and Soden 2006). Figure 5 shows the exponential rise in global-mean column water vapor in our simulations. Also shown is the global-mean and column-integrated saturation specific humidity exclud-

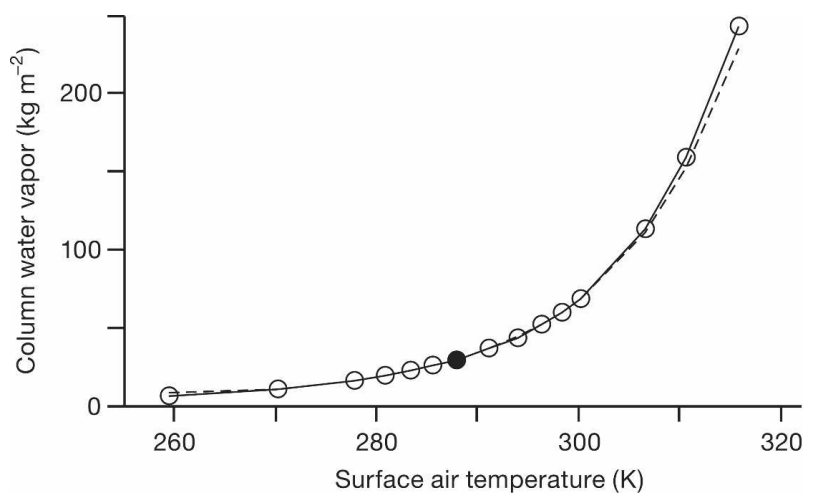

FIG. 5. Global-mean column water vapor vs global-mean surface air temperature (solid line with circles). Also shown (dashed line) is the global-mean and column-integrated saturation specific humidity, excluding contributions from levels above $\sigma=0.05$ and rescaled by a constant relative humidity factor of 0.67 so that both curves agree for the reference simulation.

ing the contribution from levels above $\sigma=0.05$ and rescaled by a constant relative humidity factor of 0.67 so that both curves agree for the reference simulation. The rate of increase at the reference climate ${ }^{3}$ is $6.2 \%$ $\mathrm{K}^{-1}$ for water vapor and $6.3 \% \mathrm{~K}^{-1}$ for saturation water vapor and the agreement is relatively good over the entire range. This is consistent with changes in water vapor content being dominated by changes in saturation specific humidity rather than changes in relative humidity, although the zonal-mean relative humidity does change as the climate changes (cf. Fig. 1).

It is widely known (e.g., Allen and Ingram 2002; Held and Soden 2006) that global-mean precipitation in global warming simulations does not increase as quickly with temperature as atmospheric water vapor content. A measure of the change in precipitation relative to water vapor content is the water vapor residence time. Figure 6 shows the residence time, defined as the global mass of atmospheric water vapor divided by the globalmean precipitation (Trenberth 1998; Roads et al. 1998; Bosilovich et al. 2005). Except for the coldest simulations, the residence time increases with surface temperature. It increases at approximately $3.8 \% \mathrm{~K}^{-1}$ at the reference simulation, consistent with the rates of increase of precipitation $\left(2.5 \% \mathrm{~K}^{-1}\right)$ and water vapor

\footnotetext{
${ }^{3}$ The fractional rate of increase of water vapor content is higher in global warming simulations if expressed relative to the water vapor content of the colder climate. The discrepancy arises because of the finite temperature change and because of the exponential dependence of water vapor on temperature. For example, interpolation of the relation between water vapor content and temperature shown in Fig. 5 suggests a fractional increase in water vapor content of $7.4 \% \mathrm{~K}^{-1}$ for a warming of $3 \mathrm{~K}$ relative to the reference climate.
} 


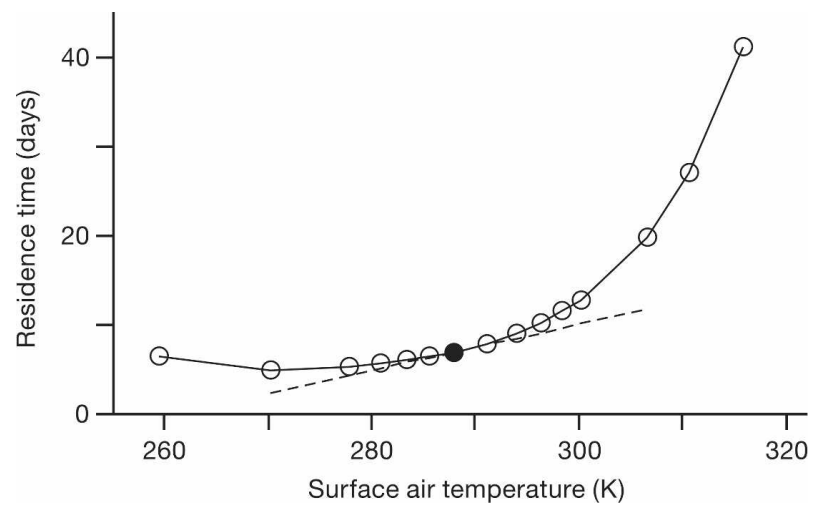

FIG. 6. Global water vapor residence time vs global-mean surface air temperature (solid line with circles). The dashed line represents a linear increase rate of $3.8 \% \mathrm{~K}^{-1}$ relative to the reference simulation.

$\left(6.2 \% \mathrm{~K}^{-1}\right)$ mentioned earlier. As discussed by Held and Soden (2006), an increase in the water vapor residence times implies a decrease in the mass exchange between the boundary layer and the free troposphere. Thus, by this measure, atmospheric circulations (but not necessarily the zonally averaged circulation) decrease in strength with increasing surface temperature over almost the entire range of climates simulated. Because the global-mean precipitation is dominated by the tropical precipitation, changes in convective mass fluxes must play an important role in allowing the global water vapor residence time to increase with temperature.

\section{Large-scale precipitation}

We analyze the precipitation associated with largescale condensation separately because the parameterization used for the large-scale condensation is relatively straightforward and is controlled by resolved motions, suggesting that the behavior of the large-scale condensation, particularly in the extratropics, may not strongly depend on model specifics. On the other hand, comparison with a select number of simulations at higher resolution reveals that the magnitude of the large-scale condensation rate is resolution dependent, with $39 \%$ greater global-mean large-scale precipitation in the reference simulation at $\mathrm{T} 85$ than at $\mathrm{T} 42$. There is a compensating decrease in precipitation associated with the convective parameterization so that the total precipitation remains almost unchanged. This indicates that there is no clear division between the parameterizations of convection and large-scale condensation, possibly as a result of resolved convection occurring at the grid scale. The fractional rate of increase of largescale precipitation with respect to temperature remains similar in the higher-resolution simulations.

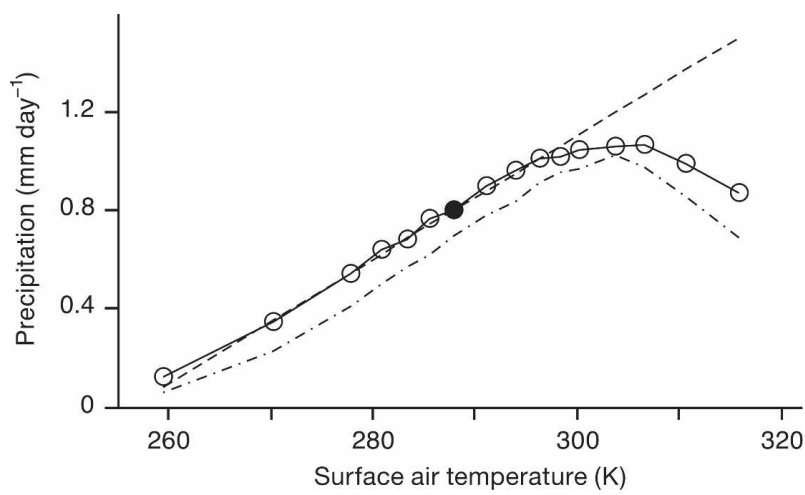

FIG. 7. Global-mean large-scale precipitation (solid line and circles) vs global-mean surface air temperature. The dash-dotted line represents large-scale precipitation according to the stochastic model result (11). The dashed line represents a linear increase of $3.2 \% \mathrm{~K}^{-1}$ relative to the reference simulation.

\section{a. Behavior of large-scale condensation and precipitation}

Figure 7 shows the global-mean large-scale precipitation. Like the total precipitation, the large-scale precipitation increases approximately linearly in temperature from the coldest simulation to the reference simulation, with a similar rate of increase of $3.2 \% \mathrm{~K}^{-1}$ at the reference simulation. The ratio of convective to largescale precipitation is, therefore, relatively constant over this range. Figure 8 shows the distribution of the mean large-scale condensation rate for the reference simulation and for the warmest simulation. In the warmer climate, most large-scale condensation occurs at higher levels in the troposphere, with some large-scale condensation remaining in the boundary layer. Significant reevaporation of falling condensate would occur in the lower troposphere in the warmer simulation if allowed for in the GCM.

To demonstrate the movement of the condensation region more generally, the movement of the center of mass of the large-scale condensation rate in the simulations is also shown in Fig. 8. The center of mass of the large-scale condensation rate $c$ for each simulation is defined by

$$
\left\{\phi_{c}, \sigma_{c}\right\}=\frac{\int_{0}^{\pi / 2} d \phi \cos \phi \int_{0}^{1} d \sigma \bar{p}_{s} \bar{c}\{\phi, \sigma\}}{\int_{0}^{\pi / 2} d \phi \cos \phi \int_{0}^{1} d \sigma \bar{p}_{s} \bar{c}} .
$$

The mean surface pressure $\bar{p}_{s}$ is the zonal, time, and interhemispheric average of the surface pressure. Otherwise, the overbar $(\bar{\cdot})$ denotes a zonal, time, and interhemispheric average on sigma levels with surface pressure weighting. 
a

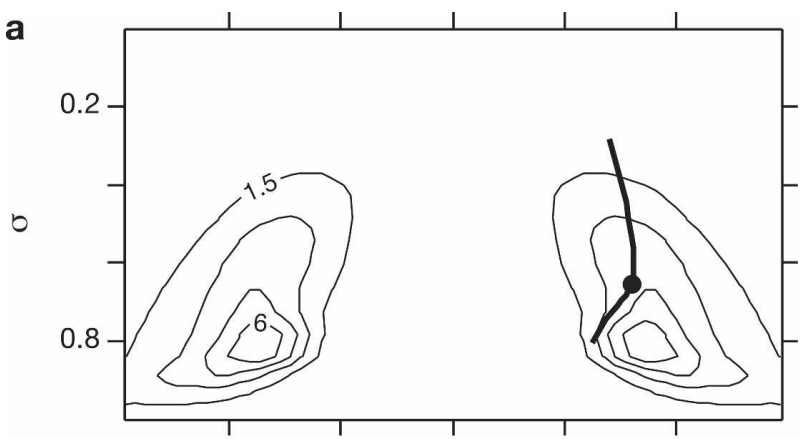

b

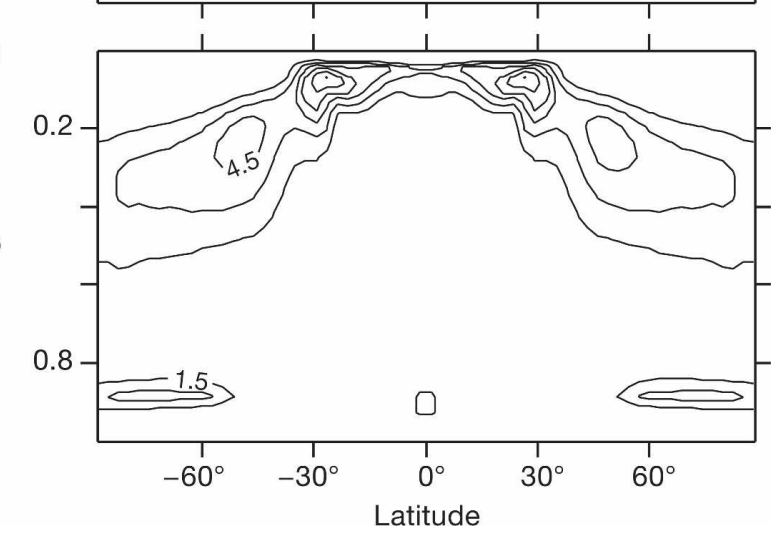

FIG. 8. Mean (zonal, time, and interhemispheric average) largescale condensation rate (contour interval $1.5 \times 10^{-9} \mathrm{~s}^{-1}$ ) for (a) the reference simulation and (b) the warmest simulation $(\alpha=$ 6.0). The thick line in the upper panel shows the movement of the center of mass (10) of the large-scale condensation rate in all simulations, with a filled circle for the reference simulation and with the center of mass moving upward from the coldest to the warmest simulation.

The center of mass of the large-scale condensation rate moves monotonically upward with increasing surface temperature. It moves poleward as the temperature increases from the coldest simulation to the simulation with global-mean surface air temperature of $294 \mathrm{~K}(\alpha=1.4)$; it moves back equatorward as surface temperatures increase further. The movement of the region of large-scale condensation to relatively colder and drier parts of the troposphere affects the largescale condensation rate, and this effect is further quantified in section $4 \mathrm{~d}$. In the earth's climate, such movement would also be associated with changes in the distribution and nature of clouds, with attendant radiative effects.

\section{b. Stochastic model}

To account for changes in the magnitude and distribution of the large-scale condensation, we use an expression for the mean large-scale condensation rate derived in O'Gorman and Schneider (2006) based on a stochastic model of moisture kinematics introduced by Pierrehumbert et al. (2007). The stochastic model is formulated using air parcels confined to a two-dimensional isentropic surface and advected by stochastic Lagrangian velocities. Each parcel carries a value of specific humidity that is reset to a local saturation value whenever the local saturation value is exceeded. In O'Gorman and Schneider 2006), we found that for Lagrangian velocities given by Ornstein-Uhlenbeck processes (Gaussian colored noise) in the limits of either very short or very long correlation times (Brownian and ballistic limits) the mean condensation rate scales as

$$
\bar{c}(y) \sim\left|\frac{\partial q_{s}}{\partial y}\right|_{\theta} \mid \sqrt{\overline{\overline{v^{\prime 2}}}} \exp \left(-\frac{d^{2}}{2 L_{e}^{2}}\right),
$$

where $\partial q_{s} /\left.\partial y\right|_{\theta}$ is the meridional gradient of saturation specific humidity along an isentropic surface, $\sqrt{{\overline{v^{\prime 2}}}^{1}}$ is the rms eddy meridional velocity, and $L_{e}$ is the rms meridional displacement of air parcels before irreversible effects become important. According to the stochastic model, condensation occurs when parcels reach saturation as they move up along an isentropic surface and cool adiabatically. The meridional distance $d$ is the distance an air parcel has to be displaced to just become saturated at the meridional coordinate $y$. Letting $\bar{q}$ denote the mean specific humidity, we set $d=y-y^{\prime}$ where the point $y^{\prime}$ is defined implicitly by $q_{s}(y)=\bar{q}\left(y^{\prime}\right)$ along an isentropic surface, so $d$ is the distance to saturation from $y^{\prime}$. The poleward decrease in saturation specific humidity along isentropic surfaces is accounted for in Eq. (11) by the isentropic gradient of saturation specific humidity. The exponential (Gaussian) factor in Eq. (11) results from the distance $d$ parcels have to travel before reaching saturation and is proportional to the probability density of a parcel displacement of this distance. By acting to cut off the condensation at certain latitudes and levels, it plays an important role in determining the region of significant large-scale condensation.

We evaluate Eq. (11) for the mean condensation rate using zonally and temporally averaged statistics from the simulations as follows. ${ }^{4}$ The stochastic model does not account for fluctuations in saturation specific humidity, so we replace $q_{s}$ with $\bar{q}_{s}$. For simplicity, we work in sigma rather than isentropic coordinates and replace the gradient along an isentropic surface with the gradient along a mean isentrope. The rms displacement $L_{e}$ is evaluated at each latitude using the potential temperature variance $\overline{\theta^{\prime 2}}$ as

\footnotetext{
${ }^{4}$ The stochastic model was formulated and tested for homogeneous velocity statistics in O'Gorman and Schneider (2006). Thus, our application of the model to the inhomogeneous velocity statistics of the GCM involves a further approximation.
} 


$$
L_{e}^{2}=\overline{\theta^{\prime 2}} /\left(\left.\frac{\partial \bar{\theta}}{\partial y}\right|_{\sigma}\right)^{2}
$$

where both the mean potential temperature gradient and variance are evaluated at $\sigma=0.84 .^{5}$ The distance to saturation $d=y-y^{\prime}$ from $y^{\prime}$ is approximated as

$$
d \simeq\left(\bar{q}_{s}-\bar{q}\right) /\left|\frac{\partial \bar{q}}{\partial y}\right|_{\bar{\theta}} \mid,
$$

where all quantities are evaluated at $y$ so that the expression (11) for the condensation rate becomes local in latitude.

We set the condensation rate in the boundary layer (below $\sigma=0.84$ ) to zero because the assumptions of the stochastic model are violated there. The use of the meridional gradient of specific humidity along a dry isentrope rather than a moist isentrope to calculate the distance to saturation in (13) is justifiable given that latent heating will only affect air parcels that have reached saturation. Use of a dry isentrope for the saturation specific humidity gradient in the expression for the condensation rate (11) is more difficult to justify in the presence of latent heating, but simple replacement of dry with moist mean isentropes in the calculation of the saturation specific humidity gradient is found to give poor results. The movement of the region of significant large-scale condensation to relatively colder parts of the troposphere in warm climates reduces the importance of the difference between dry and moist isentropes for large-scale condensation.

Figure 9 shows the condensation rate given by the analytic expression (11) for the reference simulation and for the warmest simulation and may be compared with the actual mean large-scale condensation rate in Fig. 8, with reasonable agreement except in the boundary layer and in subpolar regions.

\section{c. Movement of region of significant large-scale condensation}

Figure 9 also shows the center of mass of the condensation rate given by the analytic expression (11) and the center of mass of the actual large-scale condensation rate in the simulations, excluding contributions from the boundary layer $(\sigma \geq 0.84)$. It can be seen that the analytic expression (11) correctly captures the movement of the center of mass of the condensation

\footnotetext{
${ }^{5}$ The rms displacement $L_{e}$ can spike at latitudes (usually in the tropics) where the mean meridional potential temperature gradient at $\sigma=0.84$ changes sign. To help avoid this problem, we set $L_{e}$ to zero where the absolute value of the mean potential temperature gradient used is less than $2 \%$ of its maximum value in a given simulation.
}

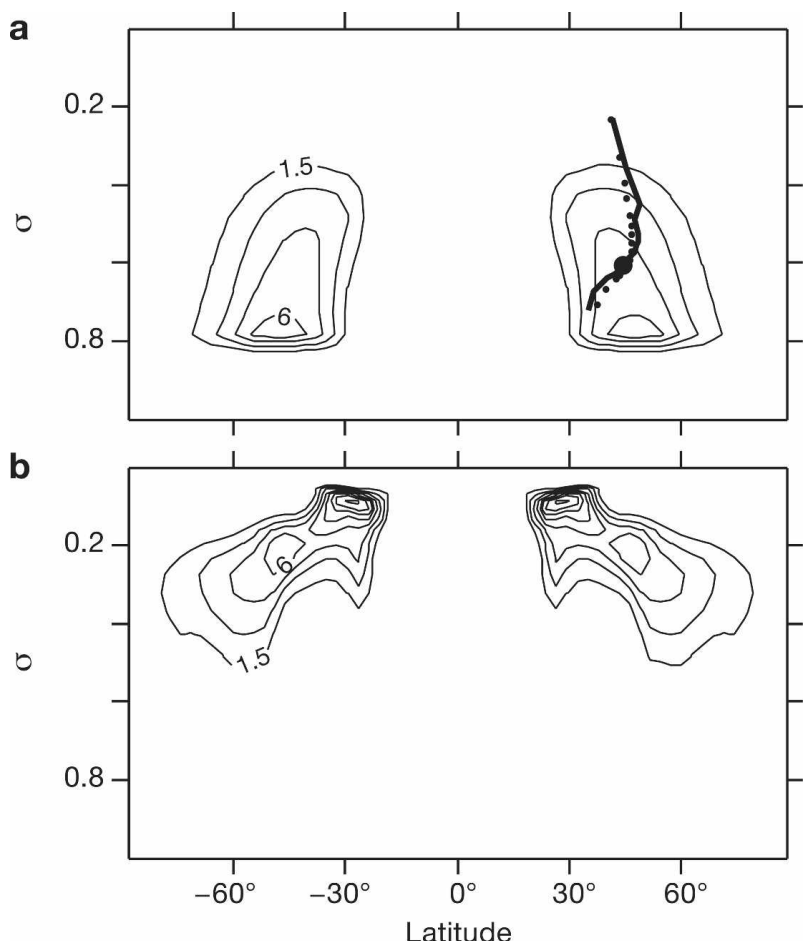

FIG. 9. Large-scale condensation rate (contour interval $1.5 \times$ $10^{-9} \mathrm{~s}^{-1}$ ) from the stochastic model result (11) for (a) the reference simulation and (b) the warmest simulation $(\alpha=6.0)$. The centers of mass of the condensation rate from the stochastic model (thick solid line and filled circle) and from the actual largescale condensation rate (dots) for all the simulations are also shown in the upper panel. Contributions from below $\sigma=0.84$ are set to zero in the calculation of the centers of mass in this figure.

rate outside the boundary layer. The increase in tropopause height with surface temperature means that at upper levels the eddy velocity and isentropic saturation specific humidity gradient in (11) increase as the climate warms. But, the upward movement of the region of significant large-scale condensation is largely accounted for by the exponential cutoff factor $\exp \left[-d^{2} /\left(2 L_{e}^{2}\right)\right]$ in (11). Figure 10 shows the value of the exponential cutoff factor at $50^{\circ}$, at $\sigma=0.7$ and at $\sigma=0.3$, for all simulations. Because the rms meridional displacement $L_{e}$ of air parcels is taken to be independent of $\sigma$, the difference in behavior at different sigma levels is due to changes in distance to saturation $d$. As the climate warms, the distance air parcels travel to become saturated increases at lower levels but decreases at higher levels, with $L_{e}$ remaining relatively constant.

Using an approximate expression for the saturation specific humidity, $q_{s} \simeq \varepsilon e_{s} / p$, where $\varepsilon=0.622$ and $e_{s}$ is the saturation vapor pressure (4), we can approximate the meridional gradient of saturation specific humidity along isentropes as 


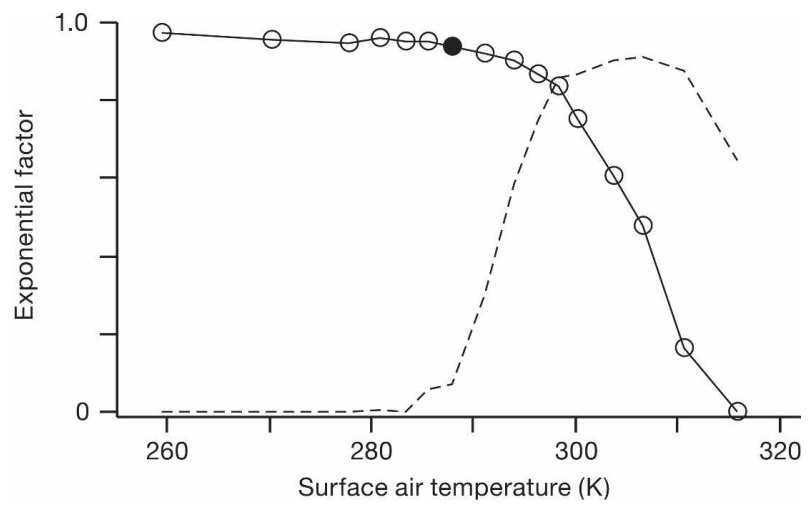

FIG. 10. Exponential cutoff factor $\exp \left[-d^{2} /\left(2 L_{e}^{2}\right)\right]$ in (11) at latitude $50^{\circ}$ vs global-mean surface air temperature, at $\sigma=0.7$ (solid line and circles) and at $\sigma=0.3$ (dashed line).

$$
\left.\left.\frac{\partial q_{s}}{\partial y}\right|_{\theta} \simeq \frac{\gamma q_{s}}{\sigma} \frac{\partial \sigma}{\partial y}\right|_{\theta},
$$

where $\gamma=\varepsilon L /\left(c_{p} T\right)-1$, and we have neglected variations in surface pressure. If the mean relative humidity $\bar{r}$ does not vary strongly, we can further approximate the distance to saturation (13) as

$$
d \sim \frac{1-\bar{r}}{\bar{r}} \frac{\sigma}{\left.\left|\frac{\partial \sigma}{\partial y}\right|_{\bar{\theta}}\right|_{\bar{\gamma}}}
$$

Changes in both the mean isentropic slope (approximated as the slope of the mean isentrope $\left.\partial \sigma /\left.\partial y\right|_{\theta} ^{-}\right)$and the mean relative humidity $\bar{r}$ contribute to changes in the distance to saturation. The isentropic slope is proportional to the meridional temperature gradient and inversely proportional to the static stability. As the climate warms, the near-surface temperature gradients generally decrease, whereas the temperature gradients at upper levels can increase. The static stability also responds differently at different levels, with decreasing static stability at upper levels as the tropopause rises with increasing surface temperature. The static stability of the extratropical troposphere does not, in general, change monotonically with increasing surface temperature in these simulations (O'Gorman and Schneider 2008). The isentropic slope in the lower troposphere $\left(\sigma=0.7, \phi=50^{\circ}\right)$ decreases by a factor of 2.6 from the reference to the warmest simulation, whereas at upper levels $\left(\sigma=0.3, \phi=50^{\circ}\right)$ it increases by a factor of 1.6. These changes in isentropic slope increase the distance to saturation in the lower troposphere and decrease it at upper levels, partially accounting for the upward movement of the condensation region.

The mean relative humidity in the extratropical lower troposphere $\left(\sigma=0.7, \phi=50^{\circ}\right)$ decreases by a factor of 1.2 from the reference to the warmest simu- lation, whereas at upper levels $\left(\sigma=0.3, \phi=50^{\circ}\right)$ it increases by a factor of 1.2 (cf. Fig. 1). The corresponding changes in $(1-\bar{r}) / \bar{r}$ in the approximate distance to saturation (15) are an increase by a factor of 1.7 in the lower troposphere and a decrease by a factor of 1.7 at upper levels, so changes in relative humidity are comparable in importance to the isentropic slope changes discussed above.

The region of large-scale condensation moves poleward as the surface temperature increases over part of the range of climates simulated. This is consistent with the poleward movement of the storm tracks, although the dependence on eddy velocity in the analytic expression (11) is not crucial for the meridional and upward movement of the region of large-scale condensation, which instead is related to changes in the mean temperature and moisture distribution. In fact, if the eddy velocity $\sqrt{\overline{v^{\prime 2}}}$ in (11) is replaced by a global-mean value, the movement of the center of mass of the largescale condensation rate remains qualitatively similar. The equatorward movement of the region of large-scale condensation for the warmest simulations is associated with the emergence of a secondary maximum in the large-scale condensation rate farther equatorward (cf. Fig. 8).

\section{d. Factors affecting mean large-scale precipitation}

The dash-dotted line in Fig. 7 shows, as a function of surface air temperature, the large-scale precipitation given by integrating the analytic expression (11). The approximately linear increase for colder climates and the peak and decrease for the warmest climates are captured. The analytic expression (11) implies that several factors affect the large-scale condensation rate. The average eddy kinetic energy associated with midlatitude transient eddies does vary in the simulations, with a maximum for climates close to the reference simulation and smaller values for much warmer or colder climates (O'Gorman and Schneider 2008). However, the condensation rate (11) scales only with the square root of the eddy kinetic energy, and the rate of change of eddy kinetic energy with respect to surface temperature is small near the reference simulation. Thus, changes in eddy kinetic energy do not account for the much lower rate of increase of large-scale precipitation compared with water vapor content.

The condensation rate (11) depends on an isentropic gradient of saturation specific humidity. The approximation (14) shows that the isentropic gradient of saturation specific humidity scales as the saturation specific humidity times the isentropic slope. The isentropic slope in the lower troposphere decreases significantly in warmer climates, an effect that tends to reduce the rate 
of increase of large-scale precipitation compared with water vapor content.

The movement of the condensation region as the climate warms leads to more condensation occurring in relatively colder and drier regions. To show that this effect is significant for climates close to the reference climate, we make a rough estimate of the changes in saturation specific humidity in the region of large-scale condensation owing to meridional and vertical movements of this region. At the reference simulation, the center of mass of the large-scale condensation rate moves upward and poleward at rates $d \sigma / d T_{s}=-0.007 \mathrm{~K}^{-1}$ and $d \phi / d T_{s}=0.3^{\circ} \mathrm{K}^{-1}$, where $T_{s}$ is the global-mean surface air temperature. The sensitivities of the saturation specific humidity at the center of mass are $-2.3 \% \mathrm{~K}^{-1}$ for vertical movement and $-1.2 \% \mathrm{~K}^{-1}$ for meridional movement, calculated as $\left(\partial \bar{q}_{s} / \partial \sigma\right)\left(d \sigma / d T_{s}\right) / \bar{q}_{s}$ and $\left(\partial \bar{q}_{s} / \partial \phi\right)\left(d \phi / d T_{s}\right) / \bar{q}_{s}$, with the partial derivatives and mean saturation specific humility evaluated at the center of mass for the reference simulation. Thus, the movement of the condensation region both vertically and meridionally is significant for the behavior of the large-scale precipitation.

This analysis suggests that the response to climate change of the large-scale precipitation associated with midlatitude weather systems depends on changes in mean isentropic slope, relative humidity, and eddy kinetic energy, in addition to changes in mean temperature.

\section{Meridional moisture flux}

The poleward flux of water vapor determines the pattern of evaporation minus precipitation $(E-P)$ and, as a latent heat flux, contributes to the energy budget of the atmosphere. The column-integrated meridional moisture flux can be written as

$$
\mathcal{F}=g^{-1} \int_{0}^{1} d \sigma \bar{p}_{s} \overline{v q},
$$

where $v$ is the meridional velocity, $q$ is the specific humidity, and $g$ is the acceleration due to gravity. Mean $\left(\mathcal{F}_{m}\right)$ and eddy $\left(\mathcal{F}_{e}\right)$ moisture fluxes can be similarly defined by decomposing the total specific humidity flux $(\overline{v q})$ into its mean $(\bar{v} \bar{q})$ and eddy $\left(\overline{v^{\prime} q^{\prime}}\right)$ components.

\section{a. Eddy moisture flux}

The meridional moisture flux in the extratropics is dominated by its eddy component. A simple scaling for the hemispherically averaged $(\tilde{*})$ and column-integrated meridional eddy moisture flux is given by

$$
\tilde{\mathcal{F}}_{e} \sim c_{e} v_{e} q_{\mathrm{ref}} \cos \phi_{s} p_{0} / g
$$

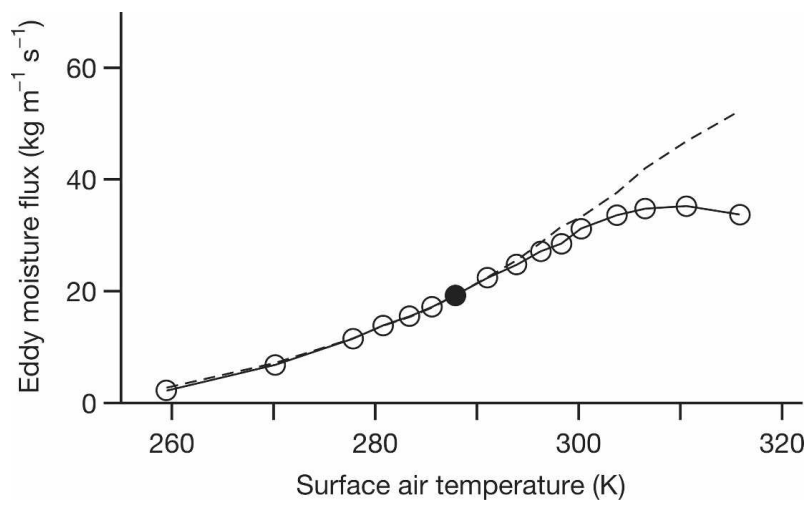

FIG. 11. Column-integrated meridional eddy moisture flux (solid line with circles) vs global-mean surface air temperature. The scaling result (17) (dashed line) is also shown with the constant $c_{e}=0.024$ chosen so that the scaling matches the eddy moisture flux at the reference simulation. The eddy moisture flux is first hemispherically antisymmetrized, then an area-weighted hemispheric average is taken. At the reference simulation, the fractional rate of increase with respect to surface temperature of the eddy moisture flux and of the scaling are $4.8 \% \mathrm{~K}^{-1}$ and $5.0 \%$ $\mathrm{K}^{-1}$, respectively.

where $c_{e}$ is a dimensionless constant, $\phi_{s}$ is the latitude of the storm track, and $q_{\text {ref }}$ is a low-latitude reference specific humidity (e.g., Pierrehumbert 2002; Caballero and Langen 2005). The eddy velocity scale $v_{e}$ is calculated as $v_{e}=\left(\mathrm{EKE} g / p_{0}\right)^{1 / 2}$, where EKE is the global mean of the eddy kinetic energy and $p_{0}=10^{5} \mathrm{~Pa}$. For the reference specific humidity $q_{\text {ref }}$, we take the mean saturation specific humidity near the top of the boundary layer $(\sigma=0.84)$ averaged over $10^{\circ}$ on either side of the equator, consistent with the meridional eddy moisture transport being dominated by filaments of water vapor leaving the tropics and subtropics (Newell et al. 1992; Zhu and Newell 1998; Schneider et al. 2006). The cosine factor in (17) accounts for the effect on the hemispherically area-averaged flux of the zonal contraction of the storm track as it moves poleward with increasing surface temperature. The latitude of the storm track $\phi_{s}$ is defined as the latitude of maximum eddy potential temperature flux $\overline{v^{\prime} \theta^{\prime}} \cos \phi$ at $\sigma=0.84$.

Figure 11 shows a comparison of the scaling (17) with the eddy moisture flux in the simulations. The agreement between the scaling (17) and the actual eddy moisture flux is good for simulations ranging from the coldest to the reference simulation. The scaling (17) is dominated by changes in the mean saturation specific humidity near the top of the tropical boundary layer $q_{\text {ref }}$, especially near the reference simulation where the eddy kinetic energy is close to a local maximum (O'Gorman and Schneider 2008). The fractional rate of increase of moisture flux with respect to surface temperature at the reference simulation $\left(4.8 \% \mathrm{~K}^{-1}\right)$ is 


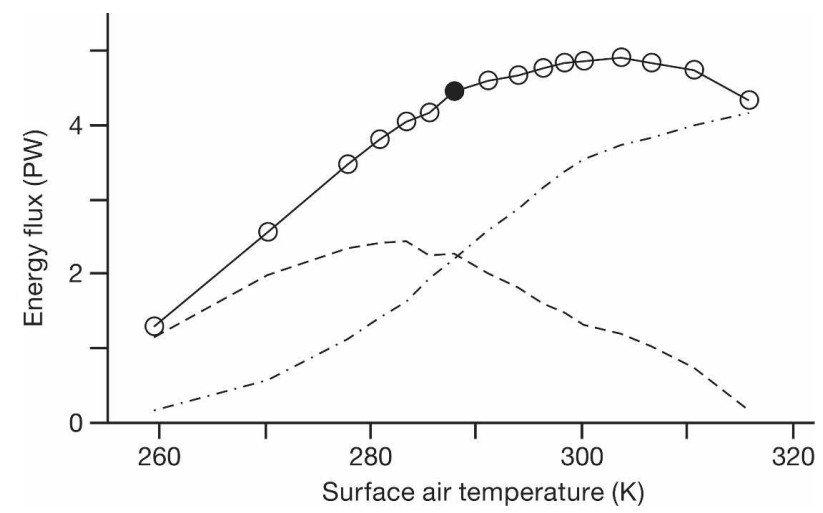

FIG. 12. Meridional energy flux (solid line and circles) at $50^{\circ}$ latitude vs global-mean surface air temperature and decomposition into dry static energy flux (dashed line) and latent heat flux (dash-dotted line).

lower than the fractional rate of increase of global water vapor content $\left(6.2 \% \mathrm{~K}^{-1}\right)$, as was also found in Held and Soden (2006) for comprehensive coupled climate simulations. The flux scaling (17) suggests that this occurs because the fractional rate of increase of water vapor near the top of the boundary layer in the tropics is smaller than that of the global-mean column water vapor. The poleward movement of the storm track also contributes to the difference between eddy moisture flux and global water vapor scaling but is less important and only leads to a rate of decrease of the cosine factor in (17) of $0.2 \% \mathrm{~K}^{-1}$ at the reference climate.

The scaling (17) overestimates the eddy moisture flux in the warmest simulations if it is taken to be correct for the reference simulation. ${ }^{6}$ One possible explanation is that the scaling of the eddy moisture flux with the lower-tropospheric tropical specific humidity is only appropriate in the colder simulations in which water vapor is more concentrated in the tropics. Alternatively, the discrepancy may arise because the mean moisture flux depends on an isentropic gradient of specific humidity (O'Gorman and Schneider 2006) and the isentropic slope becomes shallower in the warmer simulations.

\section{b. Meridional energy transport and compensation}

Since the meridional water vapor flux is a latent heat flux, its behavior has implications for the meridional energy transport. Figure 12 shows, for each simulation,

\footnotetext{
${ }^{6}$ Use of a reference specific humidity $q_{\text {ref }}$ at the edge of the Hadley cell (cf. Caballero and Langen 2005) or use of the latitude of the maximum in eddy moisture flux rather than the storm-track latitude does not improve the accuracy of the eddy moisture flux scaling and gives similar results except in the warmest simulations.
}

the meridional energy flux at $50^{\circ}$ latitude and its decomposition into dry static energy flux and latent heat flux. The latent heat flux increases with increasing surface temperature, while the dry static energy flux generally decreases with increasing surface temperature except for the coldest simulations. Although Fig. 12 shows that the energy flux attains a maximum for a certain longwave optical thickness, implying a minimum in the gradient of OLR, this does not imply a minimum in pole-to-equator surface temperature contrast: the surface temperature contrast decreases monotonically as the climate warms over the entire range of our simulations.

Several studies have found an approximate compensation between changes in dry static energy flux and latent heat flux as forcings were varied in different climate model configurations, such that the total meridional energy transport stays relatively constant (e.g., Manabe et al. 1975; Manabe and Broccoli 1985; Frierson et al. 2007). There is some compensation shown in Fig. 12 where the changes in dry static energy and latent heat flux have opposite sign, for example, near the reference simulation. Clearly, compensation is not a general feature of our simulations, and there are large changes in the total energy flux shown in Fig. 12 with corresponding changes in the distribution of OLR with latitude. The minimum in OLR as a function of latitude moves from the poles in the reference and colder simulations to the midlatitudes in the warmer simulations.

\section{Midlatitude and subtropical precipitation}

While the general pattern of mean precipitation in all simulations is similar to that in the reference simulation shown in Fig. 2, the latitudes of the subtropical minimum and extratropical maximum vary. Figure 13 shows the mean precipitation as a function of latitude and global-mean surface temperature, as well as the latitudes of the mean precipitation extrema and the latitude $\phi_{s}$ of the storm track (defined on the basis of the eddy potential temperature flux as above). The subtropical precipitation minimum moves poleward as the climate warms over most of the range of climates simulated. Similarly, the storm track moves poleward as the climate warms, as is seen in global warming simulations (e.g., Yin 2005). The latitude of the extratropical precipitation maximum is similar to and moves with the storm track latitude for climates with global-mean surface temperatures above approximately $285 \mathrm{~K}$. For colder climates, the local precipitation maximum is farther equatorward than the storm track. The nearsurface meridional temperature gradient increases significantly in colder climates, which together with the 


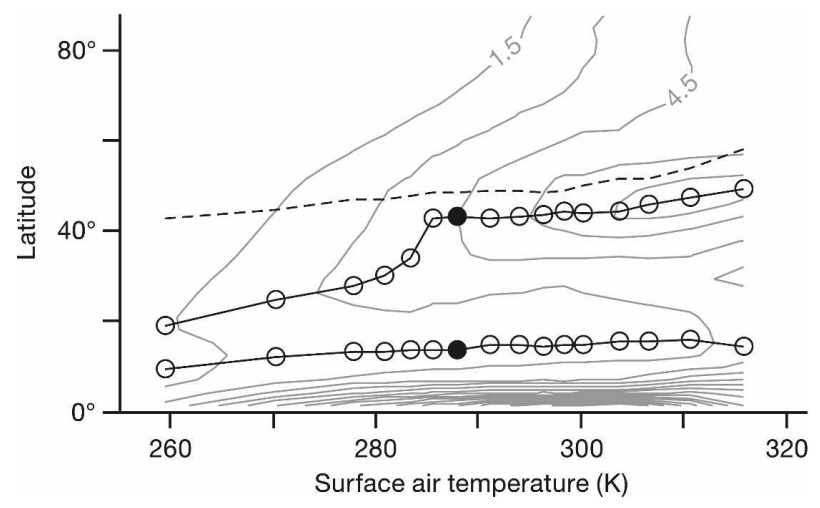

FIG. 13. Mean (zonal, time, and interhemispheric average) precipitation (gray contours, contour interval $1.5 \mathrm{~mm} \mathrm{day}^{-1}$ ) as a function of latitude and global-mean surface air temperature. Also shown are the latitudes of the subtropical minimum and extratropical maximum in mean precipitation (lower and upper solid lines with circles) and the latitude of the extratropical storm track (dashed line, see text).

nonlinearity of the Clausius-Clapeyron relation means that water vapor and evaporation are more strongly concentrated near the tropics than in warmer climates. The local maximum in large-scale precipitation stays near the storm track in all cases, consistent with largescale precipitation occurring almost exclusively in baroclinic eddies; however, the local maximum in convective precipitation stays near the storm track only for the warmest simulations.

Local precipitation at a particular latitude can be understood in terms of the column water vapor budget at equilibrium,

$$
P=E-\frac{1}{a_{e} \cos \phi} \frac{\partial}{\partial \phi}(\mathcal{F} \cos \phi),
$$

where $a_{e}$ is the earth's radius and the second term on the right-hand side is the meridional moisture flux convergence. The distribution of mean precipitation minus evaporation $(P-E)$ is of particular importance for the freshening of seawater in the oceans and runoff over land. Unlike the extratropical maximum of precipitation, the extratropical maximum of $P-E$ does not shift toward the subtropics in colder climates.

Figure 14 shows the midlatitude precipitation, measured as an average over a $6^{\circ}$ latitude band about the latitude of the storm track. The precipitation increases with increasing temperature for all but the warmest simulations. The local evaporation and convergences of the mean and eddy moisture fluxes are also plotted. The local precipitation increases both because of increasing local evaporation and increasing moisture flux convergence. The eddy moisture flux convergence is larger than the mean moisture flux convergence, and

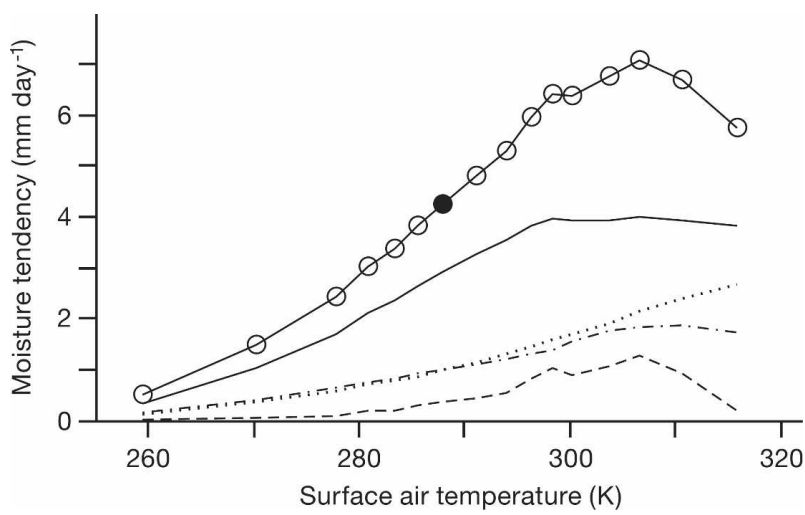

FIG. 14. Midlatitude precipitation (solid line and circles) vs global-mean surface air temperature and contributions to the column water vapor budget: evaporation (solid line), eddy moisture flux convergence (dash-dotted line), and mean moisture flux convergence (dashed line). All quantities are averaged over a $6^{\circ}$ latitude band about the latitude of the storm track. An approximate scaling (19) for the eddy moisture flux convergence is also shown (dotted line). At the reference simulation, the fractional rates of change with respect to global-mean surface temperature are precipitation $4.3 \% \mathrm{~K}^{-1}$, evaporation $4.0 \% \mathrm{~K}^{-1}$, eddy moisture flux convergence $3.3 \% \mathrm{~K}^{-1}$ and its scaling estimate $5.0 \% \mathrm{~K}^{-1}$, and mean moisture flux convergence $9.3 \% \mathrm{~K}^{-1}$.

the scaling (17) for the eddy moisture flux can also be used to predict the eddy moisture flux convergence. If we assume that the eddy flux varies over a length scale $l_{\mathcal{F}}$, the scaling (17) implies that the column-integrated eddy moisture flux convergence scales as

$$
c_{e} v_{e} q_{\mathrm{ref}} \cos \phi_{s} p_{0} /\left(g l_{\mathcal{F}}\right) \text {. }
$$

Note that we do not use a local value for the reference specific humidity but rather the same value used for the moisture flux scaling (17). Figure 14 shows the approximation (19) with the length scale $l_{\mathcal{F}}=1710 \mathrm{~km}$ taken to be constant and chosen so that the scaling matches the eddy moisture flux convergence for the reference simulation. As was the case for the eddy moisture flux scaling, the scaling (19) overestimates the rate of increase of the eddy moisture flux convergence with respect to surface temperature in warm climates.

Figure 15 shows the subtropical precipitation, measured as an average over a $6^{\circ}$ latitude band about the latitude of the local subtropical minimum in zonally and temporally averaged precipitation. Subtropical precipitation variations are more subtle because the moisture flux divergence and the evaporation have opposing effects on the magnitude of the precipitation in the subtropics (the subtropics are a net exporter of water vapor). Figure 15 shows that there is a large range of climates (including that of the reference simulation) in which the subtropical precipitation decreases slowly 


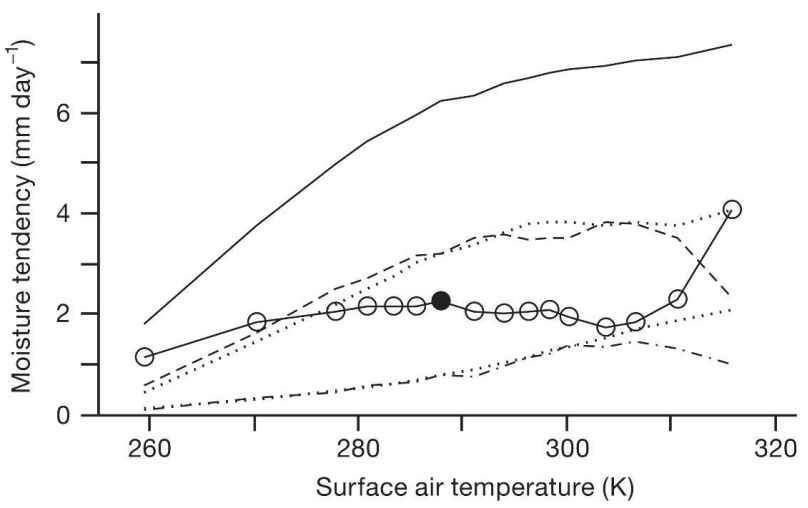

FIG. 15. Subtropical precipitation (solid line and circles) vs global-mean surface air temperature and contributions to the column water vapor budget: evaporation (solid line), eddy moisture flux divergence (dash-dotted line), and mean moisture flux divergence (dashed line). All quantities are averaged over a $6^{\circ}$ latitude band about the latitude of the subtropical precipitation minimum. Approximate scalings for the eddy moisture flux divergence (19) and mean moisture flux divergence (20) are also shown (dotted lines). At the reference simulation, the fractional rates of change with respect to global-mean surface temperature are precipitation $-0.7 \% \mathrm{~K}^{-1}$, evaporation $1.3 \% \mathrm{~K}^{-1}$, eddy moisture flux divergence $3.2 \% \mathrm{~K}^{-1}$ and its scaling estimate $5.0 \% \mathrm{~K}^{-1}$, and mean moisture flux divergence $2.1 \% \mathrm{~K}^{-1}$ and its scaling estimate $2.4 \% \mathrm{~K}^{-1}$

with increasing surface temperature. Figure 6 of Allen and Ingram (2002) shows that, in an ensemble of global warming simulations, zonal-mean precipitation in the subtropics decreases in some but not all simulations, particularly not in the Northern Hemisphere. Thus, it seems plausible that the balance between opposing changes in evaporation and moisture flux divergence shown in Fig. 15 also operates in more comprehensive GCMs.

The eddy moisture flux convergence scaling (19) can also be used as a scaling for the subtropical eddy moisture flux divergence and is shown in Fig. 15 (with a different length scale than in the midlatitudes, $l_{\mathcal{F}}=$ $2175 \mathrm{~km}$ ). Again, it is not adequate in the warmer climates. Unlike in midlatitudes, the divergence of the mean flux is the larger contribution to the total moisture flux divergence. We propose a simple scaling for the column-integrated mean moisture flux divergence,

$$
\frac{c_{m} \psi_{\max } q_{\mathrm{ref}}}{2 \pi a_{e}^{2} \phi_{\min }},
$$

where $\psi_{\max }$ is the maximum value of the mean mass streamfunction in the Hadley cell, $\phi_{\min }$ is the latitude of the subtropical precipitation minimum, and $q_{\text {ref }}$ is the mean saturation specific humidity at the top of the tropical boundary layer, as also used in the eddy moisture flux scaling (17). The dimensionless constant

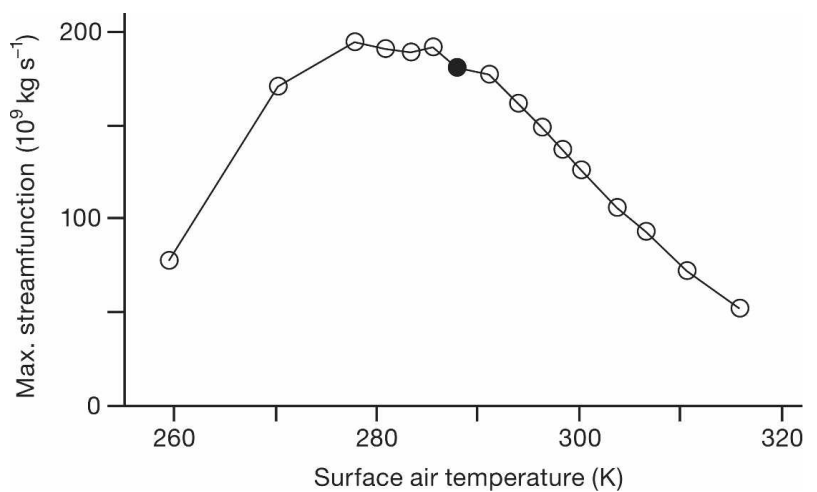

FIG. 16. Hadley cell strength (maximum value of the mean mass streamfunction in the Hadley cell) vs global-mean surface air temperature.

$c_{m}=0.80$ is chosen so that the scaling result is exact for the reference simulation. We estimate the mean moisture flux divergence in the lower troposphere since water vapor is concentrated there for all but the warmest climates simulated. The meridional mass flux and specific humidity in the lower troposphere are taken to scale as $\psi_{\text {max }} /\left(2 \pi a_{e}\right)$ and $q_{\text {ref }}$, respectively. The meridional length scale over which the divergence occurs is taken to scale like the distance from the subtropical precipitation minimum to the equator, $a_{e} \phi_{\min }$. The scaling is shown in Fig. 15 and approximately captures the behavior of the subtropical mean flux divergence. The Hadley cell strength (Fig. 16) as measured by $\psi_{\max }$ decreases with increasing temperature for climates close to the reference climate, consistent with the decrease in the strength of the Hadley cell seen in global warming simulations (e.g., Lu et al. 2007); however, for the coldest simulations the Hadley cell strengthens with increasing surface temperature (see also Fig. 1). The strong increase in $q_{\text {ref }}$ with temperature in the warmer simulations is offset in the mean moisture flux divergence scaling (20) by the increase in $\phi_{\min }$ shown in Fig. 13 and the decrease in $\psi_{\max }$ shown in Fig. 16.

It may seem surprising that the increase in water vapor export does not lead to a larger decrease in subtropical precipitation, but it is crucial to note that the water vapor export can at most equal the evaporation (in the no-precipitation limit). At the subtropical latitude of minimum precipitation in the reference simulation, evaporation is 1.6 times greater than the water vapor export, and so, although its fractional change is smaller than that of the water vapor export, changes in water vapor export and evaporation can approximately balance. Latitudes near the edge of the subtropical dry zone may see a greater decrease in precipitation as the climate warms because of the changes in the distribution of precipitation shown in Fig. 13. For example, in 
the $6^{\circ}$ latitude band about $25^{\circ}$, the precipitation rate decreases at a rate of $2.3 \% \mathrm{~K}^{-1}$ at the reference simulation.

\section{Conclusions}

We have systematically studied the large-scale mean hydrological cycle and its response to changes in longwave optical thickness. Our results show a strikingly simple relation between global-mean precipitation and temperature (Fig. 3). The relation is a combination of a linear regime and an asymptotic approach to maximum precipitation. The increase of water vapor residence time with surface temperature seen in global warming simulations is a feature of all but the coldest climates simulated here. Energy constraints are useful for explaining the qualitative behavior of global-mean precipitation but are less useful quantitatively because of significant variations in surface sensible heat fluxes. In particular, the longwave radiative loss of the atmosphere does not have the same fractional rate of change with respect to temperature at the reference simulation as the latent heating associated with precipitation. Radiative-convective simulations have a similar relation between global-mean precipitation and temperature as the full dynamical simulations, with a similar fractional rate of change of precipitation with respect to surface temperature at the reference climate. This suggests that radiative-convective simulations may be a useful tool for understanding which factors determine the rate of increase of global-mean precipitation with respect to temperature in GCMs.

Like the global-mean total precipitation, the globalmean large-scale precipitation also shows a range in which it depends approximately linearly on globalmean surface temperature. But, for the warmest simulations, the large-scale precipitation decreases with increasing temperature. The region of large-scale condensation moves upward and meridionally as the climate warms. This movement may have implications for clouds and their associated radiative forcing and for the interpretation of paleoclimate proxies based on isotopic fractionation. An analytic expression for the mean large-scale condensation rate based on a stochastic model of moisture advection and condensation relates the mean large-scale condensation rate to the mean thermal structure of the troposphere, the mean relative humidity field, and the eddy velocity variance. The analytic expression accounts for the decrease in large-scale condensation in the lower free troposphere in warmer climates in terms of a decrease in relative humidity and isentropic slope there. The analytic expression suggests that the large-scale precipitation does not scale with atmospheric water vapor content for several reasons, including changes in isentropic slope and in the region of significant large-scale condensation.

Both the subtropical precipitation minimum and the precipitation maximum in the extratropics move poleward as the climate warms. In warm climates, the extratropical precipitation maximum moves with the storm track, but for cold climates the precipitation maximum can be equatorward of the storm track, an effect related to the greater concentration of water vapor at lower latitudes in cold climates because of large near-surface meridional temperature gradients. Such changes in the distribution of precipitation are important for regional climate, particularly near the edges of the subtropical dry zones.

There are significant differences between the behavior of midlatitude and subtropical precipitation. The midlatitude precipitation increases with increasing temperature both because of increasing local evaporation and increasing meridional moisture flux convergence. By contrast, the subtropical precipitation minimum is relatively constant over a range of climates because of a balance between opposing increases in local evaporation and meridional moisture flux divergence. In this range of climates, the fractional rate of change of the moisture flux divergence is greater than that of the local evaporation, but the local evaporation is of greater magnitude than the moisture flux divergence (as it must be), so such a balance is possible.

The moisture flux convergence in midlatitudes is dominated by the eddy flux component. A simple scaling for the meridional eddy moisture flux in terms of the eddy kinetic energy, the latitude of the storm track, and a reference saturation specific humidity at the top of the tropical boundary layer is found to hold except in the warmest climates. The water vapor of the atmosphere is no longer as concentrated in the tropical lower troposphere in warmer climates, and this may explain why the scaling then overestimates the eddy moisture flux. Changes in the meridional energy flux associated with the meridional moisture flux are somewhat compensated for by changes in the dry static energy flux for a range of climates, but no general principle of compensation for the meridional energy flux applies. The moisture flux divergence in the subtropics is dominated by the divergence of the mean flux component (the flux associated with the mean meridional circulation). A simple scaling is found to hold for the divergence of the mean flux in terms of the Hadley cell strength, the distance from the equator to the subtropical precipitation minimum, and a reference saturation specific humidity at the top of the tropical boundary layer.

A general feature of our results is that there are dif- 
ferent responses of the hydrological cycle to changes in longwave optical thickness at different points in the continuum of climates states. For example, the subtropical precipitation minimum increases with temperature for the coldest and warmest simulations, but remains relatively constant in a range of climates including the reference climate. The global-mean large-scale precipitation has a maximum for a certain climate and decreases with increasing temperature for the warmest simulations. Nonmonotonic behavior is also found in the underlying dynamics, with both the eddy kinetic energy (O'Gorman and Schneider 2008) and Hadley cell strength attaining a maximum value for certain climates.

Acknowledgments. We are grateful for support by the National Science Foundation (Grant ATM0450059), the Davidow Discovery Fund, and a David and Lucile Packard Fellowship. The simulations were performed on Caltech's Division of Geological and Planetary Sciences Dell cluster. We thank Dargan Frierson for providing code for the convection and radiation schemes, Chris Walker for development of the post-processing code, and Raymond Pierrehumbert and Duane Waliser for helpful discussions.

\section{REFERENCES}

Allan, R. P., and B. J. Soden, 2007: Large discrepancy between observed and simulated precipitation trends in the ascending and descending branches of the tropical circulation. Geophys. Res. Lett., 34, L18705, doi:10.1029/2007GL031460.

Allen, M. R., and W. J. Ingram, 2002: Constraints on future changes in climate and the hydrologic cycle. Nature, 419, 224 232.

Boer, G. J., 1993: Climate change and the regulation of the surface moisture and energy budgets. Climate Dyn., 8, 225-239.

Bosilovich, M. G., S. D. Schubert, and G. K. Walker, 2005: Global changes of the water cycle intensity. J. Climate, 18, 15911608.

Broccoli, A. J., 2000: Tropical cooling at the last glacial maximum: An atmosphere-mixed layer ocean model simulation. J. Climate, 13, 951-976.

Caballero, R., and P. L. Langen, 2005: The dynamic range of poleward energy transport in an atmospheric general circulation model. Geophys. Res. Lett., 32, L02705, doi:10.1029/ 2004GL021581.

Dessler, A. E., and S. C. Sherwood, 2000: Simulations of tropical upper tropospheric humidity. J. Geophys. Res., 105, $20155-$ 20163.

Frierson, D. M. W., 2007: The dynamics of idealized convection schemes and their effect on the zonally averaged tropical circulation. J. Atmos. Sci., 64, 1959-1976.

—, I. M. Held, and P. Zurita-Gotor, 2006: A gray-radiation aquaplanet moist GCM. Part I: Static stability and eddy scale. J. Atmos. Sci., 63, 2548-2566.

$\longrightarrow,-$, and $\longrightarrow, 2007$ : A gray-radiation aquaplanet moist
GCM. Part II: Energy transports in altered climates. J. Atmos. Sci., 64, 1680-1693.

Galewsky, J., A. Sobel, and I. Held, 2005: Diagnosis of subtropical humidity dynamics using tracers of last saturation. J. Atmos. Sci., 62, 3353-3367.

Gu, G., R. F. Adler, G. J. Huffman, and S. Curtis, 2007: Tropical rainfall variability on interannual-to-interdecadal and longer time scales derived from the GPCP monthly product. $J$. Climate, 20, 4033-4046.

Held, I. M., and M. J. Suarez, 1994: A proposal for the intercomparison of the dynamical cores of atmospheric general circulation models. Bull. Amer. Meteor. Soc., 75, 1825-1830.

— , and B. J. Soden, 2000: Water vapor feedback and global warming. Annu. Rev. Energy Environ., 25, 441-475.

- and _ 2006: Robust responses of the hydrological cycle to global warming. J. Climate, 19, 5686-5699.

Hennessy, K. J., J. M. Gregory, and J. F. B. Mitchell, 1997: Changes in daily precipitation under enhanced greenhouse conditions. Climate Dyn., 13, 667-680.

Lu, J., G. A. Vecchi, and T. Reichler, 2007: Expansion of the Hadley cell under global warming. Geophys. Res. Lett., 34, L06805, doi:10.1029/2006GL028443.

Manabe, S., and R. J. Stouffer, 1980: Sensitivity of a global climate model to an increase of $\mathrm{CO}_{2}$ concentration in the atmosphere. J. Geophys. Res., 85, 5529-5554.

—, and A. J. Broccoli, 1985: The influence of continental ice sheets on the climate of an ice age. J. Geophys. Res., 90, 2167-2190.

— , K. Bryan, and M. J. Spelman, 1975: A global ocean-atmosphere climate model. Part I. The atmospheric circulation. $J$. Phys. Oceanogr., 5, 3-29.

Meehl, G. A., and Coauthors, 2007: Global climate projections. Climate Change 2007: The Physical Science Basis, S. Solomon et al., Eds., Cambridge University Press, 747-846.

Newell, R. E., N. E. Newell, Y. Zhu, and C. Scott, 1992: Tropospheric rivers?-A pilot-study. Geophys. Res. Lett., 19, 24012404.

O’Gorman, P. A., and T. Schneider, 2006: Stochastic models for the kinematics of moisture transport and condensation in homogeneous turbulent flows. J. Atmos. Sci., 63, 2992-3005.

- , and —, 2008: Energy of midlatitude transient eddies in idealized simulations of changed climates. J. Climate, in press.

Pierrehumbert, R. T., 2002: The hydrologic cycle in deep-time climate problems. Nature, 419, 191-198.

, and R. Roca, 1998: Evidence for control of Atlantic subtropical humidity by large scale advection. Geophys. Res. Lett., 25, 4537-4540.

- H. Brogniez, and R. Roca, 2007: On the relative humidity of the atmosphere. The Global Circulation of the Atmosphere, T. Schneider and A. H. Sobel, Eds., Princeton University Press, 143-185.

Rennó, N. O., K. A. Emanuel, and P. H. Stone, 1994: Radiativeconvective model with an explicit hydrologic cycle. 1 . Formulation and sensitivity to model parameters. J. Geophys. Res., 99, 14 429-14 441.

Roads, J., S. C. Chen, S. Marshall, and R. Oglesby, 1998: Atmospheric moisture cycling rate trends from model runs. GEWEX News, No. 8, International GEWEX Project Office, Silver Spring, MD, 1, 7-10.

Schneider, T., 2004: The tropopause and the thermal stratification in the extratropics of a dry atmosphere. J. Atmos. Sci., 61, 1317-1340.

— , K. L. Smith, P. A. O'Gorman, and C. C. Walker, 2006: A 
climatology of tropospheric zonal-mean water vapor fields and fluxes in isentropic coordinates. J. Climate, 19, 59185933.

Trenberth, K. E., 1998: Atmospheric moisture residence times and cycling: Implications for rainfall rates and climate change. Climatic Change, 39, 667-694.

, A. Dai, R. M. Rasmussen, and D. B. Parsons, 2003: The changing character of precipitation. Bull. Amer. Meteor. Soc., 84, 1205-1217.

Troen, I. B., and L. Mahrt, 1986: A simple model of the atmospheric boundary layer; sensitivity to surface evaporation. Bound.-Layer Meteor., 37, 129-148.

Weaver, C. P., and V. Ramanathan, 1995: Deductions from a simple climate model: Factors governing surface temperature and atmospheric thermal structure. J. Geophys. Res., 100, 11 585-11 591.

Wentz, F. J., L. Ricciardulli, K. Hilburn, and C. Mears, 2007: How much more rain will global warming bring? Science, 317, 233 235.

Xie, P., and P. A. Arkin, 1997: Global precipitation: A 17-year monthly analysis based on gauge observations, satellite estimates, and numerical model outputs. Bull. Amer. Meteor. Soc., 78, 2539-2558.

Yin, J. H., 2005: A consistent poleward shift of the storm tracks in simulations of 21 st century climate. Geophys. Res. Lett., 32, L18701, doi:10.1029/2005GL023684.

Zhu, Y., and R. E. Newell, 1998: A proposed algorithm for moisture fluxes from atmospheric rivers. Mon. Wea. Rev., 126, $725-735$. 